Derecho \& Realidad

Vol. 13 - Núm. 25 •Enero-Junio de 2015

Págs. 111-130 • ISSN: 1692-3936

\title{
Participación indígena a nivel internacional $y$ afectación al estatocentrismo en la comunidad internacional*
}

\author{
Indigenous participation at international level \\ and disturbance to the state centrism in the \\ international community
}

\author{
Asier Tapia Gutiérrez** \\ asiertg@hotmail.com
}

\section{Resumen}

El artículo expone la participación de los pueblos indígenas a lo largo de las últimas décadas y de qué manera esta ha influido en el estatocentrismo sustentador del sistema internacional de derecho internacional. Con este objeto se han analizado analogías con otro tipo de grupos que han participado en la elaboración de instrumentos internacionales, para posteriormente estudiar las motivaciones de la participación de los pueblos indígenas y ver de qué forma guardan relación con la soberanía indígena. También se exponen los conceptos elementales que respaldan la participación indígena así como su

Fecha de aceptación: 30 de julio de 2014

Fecha de recepción: 03 de septiembre de 2014

Fecha de aprobación final: 28 de febrero de 2015

* Artículo de reflexión, producto de la tesis doctoral del autor, titulada: Empresa y Derechos Humanos en Colombia. Confluencia entre los modelos de Desarrollo y Derechos Humanos.

** Doctorando Universidad de Deusto. Profesor Relaciones Internacionales Universidad Tecnológica de Bolívar. 
soberanía y cómo guardan relación con la subjetividad jurídica internacional. Antes de mostrar las conclusiones se presentan los resultados prácticos de la investigación en términos de institucionalidad así como la forma en que afectan al estatocentrismo.

\section{Palabras clave}

Pueblos indígenas, estatocentrismo, participación internacional, derecho internacional.

\section{Abstract \\ The article exposes the indigenous participation along the last decades, and its influence on the state centrism, which is the sustainer of the international law system. For this purpose we have studied analogies with other groups that have participated in the elaboration of international instruments. Thereafter we analyze the motivations of the participation of indigenous peoples and how this are related to indigenous sovereignty. We also expose the concepts in which indigenous participation and indigenous sovereignty are based on and their relation to international legal subjectivity. Before displaying the conclusions, the practical results of the research are presented in terms of institutionalism and how they could affect state centrism.}

\section{Keywords}

Indigenous peoples, international law, state centrism, international participation. 


\section{Introducción}

El artículo analiza el modo en que la participación de los pueblos indígenas ha influido y afectado al estatocentrismo del sistema internacional de Estados y más concretamente al sistema internacional de derecho internacional.

Para analizar esta afectación, en el primer capítulo se hace una comparación de la participación a nivel internacional de otros grupos o individuos (personas en situación de discapacidad, familiares de personas desaparecidas), que pueda ser comprendida a priori como análoga para dilucidar si efectivamente las motivaciones y objetivos hacen comparables sus participaciones con los de los pueblos indígenas. La afirmación de esta similitud supondría por tanto una debilidad para las teorías que afirman un ataque claro al estatocentrismo del sistema y en especial a quienes llegan a equiparar la participación indígena con la estatal en el sistema internacional. Por el contrario, afirmar unas diferencias más o menos importantes respecto de los otros grupos con que se compara, supondría al menos un fortalecimiento en términos jurídico-políticos de la especificidad de la participación de los pueblos indígenas en clave de soberanía.

En el segundo capítulo se exponen algunas de las motivaciones de la participación de los pueblos indígenas en las instituciones del sistema internacional, a fin de evidenciar si existe un intento de confrontar el estatocentrismo del sistema, si simplemente existe un deseo de ver mejoradas sus condiciones de vida, o si el objetivo principal de esta participación es lograr un cambio sistémico en términos de aceptación de sus cosmovisiones, que indirectamente pueda propiciar una afectación al estatocentrismo así como una mejora de sus condiciones de vida acorde a sus cosmovisiones.

Parte importante en este análisis supone tomar como referencia el porqué los pueblos indígenas han podido participar en el sistema: si debido a las fuerzas propias que han impedido a los gobiernos contener el empuje o si bien han sido adecuadamente canalizados por parte del propio sistema para disminuir otro tipo de protestas a otros niveles que pudieran provocar mayores disturbios escapando de algún modo de la influencia de los gobiernos respectivos. Esta exposición de las facultades que han permitido la participación se irá comentando a lo largo de todo el artículo, si bien con un mayor énfasis y concreción en este apartado segundo.

En el tercer capítulo se estudia la participación indígena en términos de logros relativos al reconocimiento de los conceptos "pueblo" y "derecho de autodeterminación", ya que estos conceptos suponen la sustancia misma que pudiera derivar en la consecución de los objetivos indígenas respecto de sus reivindicaciones 
históricas, así como respecto de la posible afectación que pudiera sufrir el estatocentrismo al atacarse el sistema de Estados-nación emanado de la Paz de Westfalia que con el tiempo implicó la creación de un sistema internacional con esos Estados-nación como mandantes del mismo.

En un cuarto capítulo se expone brevemente un debate en términos de conclusiones mostrando de qué manera lo presentado hasta ese momento implica o no, que los pueblos indígenas puedan ser considerados sujetos de derecho al mismo nivel que los Estados, a un nivel intermedio, o a un nivel equivalente a otros sujetos de derecho, y qué supone esto para la soberanía indígena y, por ende, hacia el propio sistema.

En el último de los capítulos, antes de las conclusiones finales, se estudiará qué supone la conformación de dos instrumentos, cuando menos peculiares sino innovadores, para el sistema internacional como son el Foro Permanente para las Cuestiones Indígenas y el Mecanismo de Expertos sobre Derechos de los Pueblos Indígenas. El estudio se realizará en directa relación con los dos conceptos expuestos en el apartado anterior, para extraer conclusiones de unos logros emanados de la participación progresiva de los pueblos indígenas en diversos órganos del sistema y que a su vez implicaron la creación y una participación con visos de permanencia en los mismos. En este apartado se realizará un avance de las conclusiones sobre si el logro de la conformación de estos dos instrumentos se ciega en una participación en cierto modo irrelevante comprendida como un gran objetivo por parte de los pueblos indígenas, o si hay previsiones prospectivas de mayores logros fundados en estas dos instituciones.

\section{Comparación entre diversos actores que participan en la discusión y elaboración de instrumentos internacionales que los afectan}

Uno de los mayores argumentos de los defensores de la vinculatoriedad de la Declaración de Derechos de los Pueblos Indígenas (UN, 2007), (en adelante Declaración) consiste en defenderla como un tratado entre pueblos y Estados en términos de igualdad, pese a ser evidente que el terreno en que se disputaba esta negociación es el dibujado por la sociedad occidental y su cosmovisión hegemónica a nivel internacional.

Para defender esta posición negociadora de igual a igual, uno de los fundamentos básicos se sostiene en la primigenia participación de los pueblos indígenas en el instrumento internacional (la ya citada Declaración) que iba a suponer un reconocimiento específico de sus derechos colectivos, más allá de que ello supusiera la creación-reconocimiento de unos derechos humanos nuevos o bien un desarrollo 
adecuado de los ya proclamados en la Declaración Universal de Derechos Humanos ${ }^{1}$. Sin embargo, hay autores como Luis Rodríguez-Piñero que afirman que esta participación no es primigenia, ya que hay otras participaciones análogas de personas o grupos de personas en el desarrollo de instrumentos de los que ellos mismos iban a ser objeto con su aprobación. De acuerdo con estos autores el aumento cuantitativo de grupos que participan en la institucionalidad internacional formaría parte de un proceso más amplio de cambio en el ámbito internacional, conocido como humanización del mismo (Meron, 2000, p. 239-278).

Entre ellos se puede destacar cómo en la Convención sobre los Derechos de las Personas con Discapacidad se ha procedido a incentivar la participación de personas en esta situación, especialmente por las ONG de países pobres, tal y como ocurrió también con la Convención Internacional para la Protección de todas las Personas contra las Desapariciones Forzadas y la participación de sus familiares en lo que se concibe como un proceso de dar mayor participación a las víctimas o beneficiarios de los futuros estándares de derechos humanos en las negociaciones de los Instrumentos respectivos (ICHRP, 2006, p. 42 y sig.).

En lo que respecta a la comparación entre la participación de los pueblos indígenas y las personas en situación de discapacidad y sus motivos y objetivos, existen algunas similitudes pero también algunas diferencias importantes. Entre las similitudes es digno destacar cómo ambos participan para evitar ser objeto de políticas asistencialistas y poder ser sujetos activos de las mismas, con la característica sustantiva de que el asistencialismo hacia los pueblos indígenas era además tiznado de políticas integracionistas que pretendían el fin de sus particularidades culturales e identitarias. La diferencia fundamental es que la participación de los pueblos indígenas tiene como objetivo seguir manteniendo una cosmovisión distinta a la hegemónica propugnada habitualmente desde los mismos instrumentos internacionales de derechos humanos. Asimismo a menudo ha habido dudas de si el problema con las personas en situación de discapacidad era tanto un problema de derechos humanos como de inclusión social y en la Convención se observa cómo algunas de las soluciones van dirigidas no tanto a los individuos como a la sociedad en su conjunto, ya que como afirman Agustina Palacios y Francisco Bariffi el objetivo es "que las personas con discapacidad puedan tener iguales oportunidades que el resto de personas en el diseño y desarrollo de sus propios planes de vida" (2007, p. 24).

En lo que respecta a la participación de asociaciones de familiares de personas desaparecidas en la Convención que trata esta materia, el objetivo principal es lograr un instrumento que impida que este tipo de delito se repita. Las diferencias

\footnotetext{
Una discusión entre estudiosos más pragmáticos como puedan ser el relator Anaya y otros más puristas entre los que se destaca la posición de Bartolomé Clavero.
} 
con la participación indígena son totales, tanto en lo que respecta a los motivos que facultan la participación como a los objetivos de la misma.

Al observar la participación y la lucha de la mujer (o el hombre por la igualdad de derechos de ambos) es sustancial cómo en cierto modo, al igual que los pueblos indígenas, atacan el corazón de los derechos humanos. La mujer lo hace esencialmente para confrontar una visión androcéntrica de los mismos que provocaban y provocan una asimilación de lo humano con lo masculino, propiciada fundamentalmente por una historia de participación durante todas las fases de negociación y acuerdo de los órganos e instrumentos de derecho internacional esencialmente masculina. Con esta participación se intenta que la mujer se erija en sujeto de derecho para lograr una igualdad de género, pero a diferencia de los indígenas, la mujer no ha contradicho la comprensión individual e individualista de los derechos humanos, que posibilite conjuntamente los "pueblos" como sujeto colectivo de derecho. Asimismo, de modo alguno hace referencia a la posición de los Estados en cuanto sujeto casi-único de poder en el sistema internacional que de forma directa o indirecta está retando la participación indígena.

\section{2. ¿Qué motiva la participación indígena en la sociedad internacional?}

La motivación de la participación de los pueblos indígenas en la institucionalidad internacional puede ser analizada desde diferentes puntos de vista y es factible que no haya una única posición entre la gran cantidad diversa y creciente de participación de estos pueblos en la sociedad internacional.

Desde el punto de vista de muchos pueblos indígenas, la motivación y el objetivo puede ser lograr un reconocimiento como pueblos que posibilite una reparación histórica de los daños sufridos desde siglos atrás ${ }^{2}$. La tesis del reconocimiento como reparación puede conjugarse con los derechos sucesivos (de desarrollo del derecho de autodeterminación, núcleo pendular de todos ellos) que pueden lograr a partir de este reconocimiento como pueblo en los diversos mecanismos internacionales de derechos humanos. Otros pueden tener como objetivo retar en términos de poder a la sociedad internacional y al Estado como actor preponderante, si bien estudiando su participación histórica es difícilmente defendible esta posición, aunque los obstáculos que los Estados a menudo imponen en la esfera internacional reflejen un miedo a esta posibilidad.

Ello no obsta sino más bien explica que en su lucha reparadora, los pueblos indígenas hayan facultado la posibilidad de cambiar el sistema internacional para que se

2 Al respecto de las diversas medidas de reparación a los pueblos indígenas, véase Gómez (2009, p.157-193). 
adecúe a la perspectiva cosmovisional propia de estos pueblos que no ha sido reflejada en unos derechos humanos dirigidos por el individualismo de origen occidental. De esta manera se ve reflejada la "primera ironía" de Patrick Glenn (2011, p. 171-183), emanada pero perfeccionada del artículo de Adam Kuper (2003, p. 389-402), donde muestra con cierto tono crítico cómo los pueblos indígenas demandan reconocimiento para formas alternativas de comprender el mundo, realizando estas demandas en un lenguaje de la teoría cultural occidental. Cuestión que no hace sino reflejar la capacidad de adaptación de los pueblos indígenas para el logro de sus objetivos, a menudo resaltada por el relator Anaya como la explicación fundamental a los avances de la lucha indígena. En este sentido, Stephen Allen (2011, p. 248) destaca cómo en los pueblos indígenas el declinar operativo del Estado es una narrativa que usan para que la lucha a nivel internacional se refleje en los diferentes espacios nacionales en que cada pueblo indígena participa, así como para promover su versión paralela de soberanía indígena.

Ello alimenta la cuestión del porqué la sociedad internacional permite e incluso alienta esta participación que puede socavar la estructura de funcionamiento de un sistema institucional internacional eminentemente estatocéntrico. La respuesta que buena parte de los académicos damos a este hecho es la existencia de un deseo de cooptar el movimiento indígena internacional. No obstante, el sentimiento de culpa como promotor y sustento de la colonización por los Estados creadores del mismo sistema ha supuesto también un elemento sustancial de esta laxitud e incluso proactividad ante la participación indígena. Esta cuestión es más destacable si la comparamos con la obstaculización a la participación que se les ha impuesto a otros grupos con algunas características comunes, como pudieran ser las minorías u otros grupos no estatales.

La fuente motivacional más importante que alimentó y permitió la resistencia durante la época de las sucesivas conquistas y que con el advenimiento de la globalización ha permitido una mayor interrelación a mayor velocidad entre los diversos pueblos indígenas, no solo latinoamericanos sino también de otros continentes, ha sido el deseo de mantener estas cosmovisiones propias que, en la mayoría de los casos, chocan con el sistema de desarrollo impuesto por los Estados a los que históricamente han sido tratados de integrar forzosamente en términos de "civilización". Esto se aprecia de manera fundamental en relación con la política extractivista y la permanente lucha indígena por el consentimiento previo libre e informado en relación con la misma.

Si bien la gran cantidad de pueblos indígenas existentes en el mundo y la diversidad entre ellos, no solo cosmovisional sino estratégica en términos de espacio y tiempo, dificulta enormemente generalizar cuál sea el motivo del paso de la lucha desde el nivel local, regional o estatal hasta el ámbito internacional, es evidente que existe 
un conjunto de motivos comunes a todos o buena parte de ellos. Entre ellos se pueden destacar las estadísticas de pobreza que los sitúan siempre en el punto más bajo entre los excluidos del sistema ${ }^{3}$, que no suponen sino un acicate más para el fomento de la lucha, así como una evidencia más de que el sistema de desarrollo que les impone la sociedad occidental no se adapta adecuadamente a sus intereses cosmovisionales. Empero, como afirma Salvador Martí respecto de los pueblos indígenas "obviamente la opresión y la pobreza, por sí solas, no presuponen la aparición de ciclos de acción colectiva ni la emergencia de nuevos actores" (2007, p. 127-149).

Otro de los motivos elementales es sin duda la imposibilidad de lograr los objetivos en el nivel estatal, tal y como le ocurrió al considerado primer indígena que dio el paso al espacio internacional, Kayuga Deskaheh ${ }^{4}$. La perentoriedad de salir para lograr estos objetivos se sumó a unas nuevas posibilidades de expandir la lucha de un sistema que por un lado les complica la existencia y por otro les concede beneficios (Brysk, 2007, p.17-31), como puede ser la mejora de las comunicaciones con las nuevas tecnologías o el apoyo por parte de asociaciones temáticas relacionadas con su causa, entre otros. Todo ello posibilitó progresivamente la participación y el cambio de perspectiva de un poscolonialismo integrador y asimilacionista a un multiculturalismo que pudiera derivar en la interculturalidad.

Como vamos a ver posteriormente cuando analicemos las temáticas abordadas en los organismos indígenas internacionales, el gran reto de los pueblos indígenas a nivel internacional es ver reconocido el carácter colectivo de su existencia en términos de derechos, para posteriormente tratar de que los Estados en que habitan reflejen en la medida de lo posible estas características especiales en la legislación interna, que es la que definitivamente les permite o no coartar la vida acorde a sus cosmovisiones.

El objetivo de reparación que exponíamos más arriba sería por tanto un objetivo secundario pero interrelacionado con el del reconocimiento como sujeto colectivo y comprendiendo la reparación como punto final a las sucesivas teorías de asimilación e integración impuestas de manera continúa por los estados así como por los organismos internacionales. Y el reconocimiento sería a su vez un medio para lograr instrumentos de reparación varios en términos de derechos.

Es curioso sin embargo que uno de los todavía grandes instrumentos y el único vinculante para la defensa de los derechos indígenas como es el Convenio 169 de la

\footnotetext{
3 Sobre comparación de pobreza en grupos indígenas y no indígenas en América Latina, véanse Damman (2010) y Rensahw \& Wray (2004).

$4 \quad$ Véase historia de la participación indígena en UN (2008).
} 
Organización Internacional del trabajo (OIT) en 1989 fuera elaborado sin participación directa de los pueblos indígenas (Rodríguez-Piñero, 2005). No obstante, una de las peculiaridades del método de trabajo en esta organización es la importancia de la cooperación entre gobiernos y organizaciones de trabajadores y empleadores que, sumada a un cambio de perspectiva en la sociedad internacional con una creciente influencia de los pueblos indígenas a través de organizaciones de la denominada sociedad civil global, supuso un cambio sustancial en la expresión jurídica concerniente a los pueblos indígenas en el ámbito internacional.

\section{Pueblos indígenas y derecho de autodeterminación: dos conceptos controvertidos}

Uno de los elementos que en todo momento ha sido uno de los grandes avatares de la lucha indígena ha sido la obtención del reconocimiento como pueblos. Ello debería conllevar la capacidad de una autodeterminación equivalente a la expresada en los artículos primeros de los Pactos Internacionales de Derechos Civiles y Políticos y Económicos Sociales y Culturales de 1966. Sin embargo, en todas las apariciones del concepto hasta el momento se ha limitado de una u otra manera la equiparación con estos Pactos, dejando solamente el poder del concepto en términos de reconocimiento como medida de reparación histórica.

El reconocimiento del término pueblo supondría en principio un bastión fundamental para el reconocimiento indígena, pero simultáneamente podría suponer un ataque a la estructura estatocéntrica del sistema internacional que defiende férreamente la soberanía de sus Estados miembros en tanto en cuanto sean reconocidos como tal por el resto de Estados de la sociedad internacional. Un reconocimiento ex aequo del derecho de autodeterminación supondría un reconocimiento simultáneo del resto de derechos indígenas en la medida en que todos son la fundamentación práctica del anterior.

Dentro del propio indigenismo y del academicismo relativo a los pueblos indígenas ha existido siempre la dificultad de lograr un concepto de consenso en relación con el término pueblos. Esta dificultad es alentada por los Estados ante las peligrosas consecuencias que pudiera tener este reconocimiento. Los pueblos indígenas siempre se han mantenido en que la autoidentificación como pueblos debe ser el elemento fundamental para que en el ámbito internacional sean reconocidos como tal, ya que como expresa el profesor Gonzalo Aguilar (2006, p. 106-119), ello forma parte de un reconocimiento "individual de determinación de su propia identidad cultural reconociéndose en aquélla como del grupo". Ello puede estar implicando indirectamente que ante la imposibilidad de avances jurídicos en las instituciones internacionales por parte de las minorías, algunos miembros pertenecientes a estos grupos estén adoptando la etiqueta de pueblos indígenas lo que "probablemente 
lleve al colapso del sistema internacional de derechos indígenas" (Kymlicka, 2011, p. 206), con el fin de beneficiarse de algunos de los derechos que también son parte de sus reivindicaciones.

\subsection{El concepto pueblos desde el Convenio 169 de la OIT a la Declaración de Derechos Indígenas}

Uno de los primeros estudios en profundidad con relación al concepto pueblo (en realidad poblaciones) que le dio la importancia debida fue sin duda el extenso estudio del ecuatoriano Martínez Cobo y que hacía referencia a (1) descendientes de quienes habitaron sus territorios y fueron conquistados o se instalaron desde el exterior, (2) -doctrina del agua azul-, que ahora viven acorde a sus particulares costumbres y tradiciones sociales, económicas y culturales, (3) bajo un Estado que incorpora primordialmente las características sociales, culturales y nacionales de la población mayoritaria (4). Es destacable que a lo largo del estudio se expresa que no hay una necesidad de conquista para considerarse indígena (Minde, 2007, p. 15 y sig.). Esta definición desde luego no supone ningún atentado ni ataque por mínimo que resulte contra la soberanía estatal al no aparecer siquiera el concepto pueblos. Asimismo tampoco hace referencia alguna a la autoidentificación, pero sí evidencia la cercanía del mismo con la colonización en los dos primeros puntos expresados mientras que en los dos siguientes se refieren sintéticamente a los motivos del porqué los pueblos indígenas luchan por la autodeterminación. Debe ser tenido en cuenta que este informe data del año 1972 y se atisba el proceso de cambio que se afianzaría principalmente en 1989 con el Convenio 169 de la OIT $^{5}$ en relación con la postura frente a las cosmovisiones indígenas, resultando plenamente acorde con el interés de los Estados miembros.

El Convenio 169 de la OIT que no es sino una revisión del Convenio 107 de la misma organización, incluye por vez primera el término pueblos indígenas y tribales a diferencia del anterior que hablaba de poblaciones ${ }^{6}$. Ello supuso un primer paso importante no exento de polémica, ya que hubo el temor de una posible "desintegración de muchos de los estados miembros y al debilitamiento de su unidad nacional"7. Como dijimos previamente no existe una participación directa indígena en su discusión pero es evidente que tanto en lo que respecta al término pueblos como en el resto del contenido se acerca sustancialmente a las reivindicaciones indígenas históricas. En su artículo número 1 se expone el concepto de a qué pueblos va dirigido el citado Convenio:

\footnotetext{
5 "Informe sobre el problema de la discriminación contra las Poblaciones Indígenas", Conclusiones, propuestas y recomendaciones, E/CN. Sub.2/1986/7add4.

La influencia de la OIT al respecto del término pueblos, en Erueti (2011, p-93-121).

P.19 VI-2 Memoria de la OIT sobre revisión del Convenio 107, p. 19.
} 
A. A los pueblos tribales en países independientes, cuyas condiciones sociales, culturales y económicas les distingan de otros sectores de la colectividad nacional, y que estén regidos por total o parcialmente por sus propias costumbres o tradiciones o por una legislación especial;

B. A los pueblos en países independientes, considerados indígenas por el hecho de descender de poblaciones que habitaban en el país o en una región geográfica a la que pertenece el país en la época de la conquista o la colonización o del establecimiento de las actuales fronteras estatales y que, cualquiera que sea su situación jurídica, conservan todas sus propias instituciones sociales, económicas, culturales y políticas o parte de ellas.

2. La conciencia de su identidad indígena o tribal deberá considerarse un criterio fundamental para determinar los grupos a los que se aplican las disposiciones del presente Convenio.

3. La utilización del término "pueblos" en este Convenio no deberá interpretarse en el sentido de que tenga implicación alguna en lo que atañe a los derechos que pueda conferirse a dicho término en el derecho internacional ${ }^{8}$.

Podemos observar un contenido bastante similar al previamente expuesto por el informe Martínez Cobo al contener la referencia bastante clara a la colonización, así como el mantenimiento de la diferenciación institucional y de comprensión, social, cultural o económica, lo que podríamos definir en el mantenimiento de cosmovisiones propias. El gran cambio es la ausencia en el citado informe de la conciencia de identidad indígena que exponíamos antes con el nombre de autoidentificación, como criterio fundamental para que sean aplicadas las disposiciones del Convenio 169.

Como colofón al artículo, el subpunto 3 pone en evidencia los miedos estatales referidos previamente arrebatando la capacidad jurídica al término "pueblos", al impedir su equiparación con los Tratados de 1966. Ello les suponía a los pueblos indígenas una victoria en términos de su deseo de reconocimiento como sujetos colectivos de derechos, corroborado posteriormente en diversas partes del mismo Convenio ${ }^{9}$; sin embargo una limitación extraordinaria en términos de ejercicio del derecho de autodeterminación, que como dijimos previamente es el derecho colectivo sobre el que se sustentan todos los demás.

En lo que a la Declaración respecta, una de las cuestiones que puede llamar la atención del contenido es que el término pueblos indígenas aparece en numerosos artículos de su contenido, si bien en ninguno acompaña explicación de qué o quiénes

Artículo 1 del Convenio 169 de la OIT sobre Pueblos Indígenas y Tribales en Países Independientes. 9 Al respecto profundizar en Gómez (2006, p.133-153). 
son estos pueblos, cuáles son los requisitos para que estos pueblos puedan ampararse en el contenido de la Declaración, en definitiva a quién va dirigida concretamente, por tanto, esta Declaración. Los diversos pueblos indígenas durante su proceso de elaboración y discusión insistieron en que salvo la inclusión del adjetivo "indígenas", el artículo era calcado del existente en los Pactos Internacionales de 1966, con el objetivo de que la aplicación del derecho de autodeterminación no sufriera menoscabo alguno al referido en estos pactos (Kempf, 2009, p. 51-65). Existe también una "cláusula" similar al artículo 1.3 del Convenio 169 de la OIT, en la Declaración en su artículo 46 cuando afirma que nada de lo contenido en la presente Declaración "se entenderá en el sentido de que autoriza o alienta acción alguna encaminada a quebrantar o menoscabar, total o parcialmente, la integridad territorial o política de Estados soberanos e independientes" ${ }^{10}$. El artículo sostiene la imposibilidad de usar el término pueblos y el consiguiente derecho a la libre determinación, como llave para un hipotético derecho a la secesión, manteniendo -en apariencia, al menos- íntegro, el derecho de autodeterminación.

Aceptar que no se incluya una conceptualización del término "pueblos indígenas" supone un vacío pragmático derivado de que tras casi veinte años de discusiones, la imposibilidad de lograr un acuerdo sobre este asunto supusiera un obstáculo que acabara con los esfuerzos llevados a cabo por todas las partes. Por parte de los pueblos indígenas se puede realizar la lectura de que es un reconocimiento de su siempre prioritario requisito de autoidentificación. No obstante, en una lectura puramente realista de esta ausencia, es innegable que en un sistema internacional comandado aún por los Estados, los pueblos indígenas que todavía no hayan sido reconocidos en cuanto tales por los Estados de los que voluntariamente o no forman parte, no van a obtener beneficio alguno para sus reivindicaciones en términos de logro de reconocimiento más allá del que tuvieran antes de la votación de esta Declaración (Tapia, 2010) e incluso puede darse el caso de verse alejados de los denominados caucus indígenas internacionales. La aceptación por parte indígena se puede comprender además por el pragmatismo previamente expuesto de no ver socavado todo el trabajo y avances logrados durante veinte años, como un modo de saciar sus ansias de reconocimiento en cuanto tales, aun sabedores de que este pueda no ser completo o estar sometido a los avatares propios de un sistema para ellos extraño hasta poco tiempo atrás. Evidencia además que la secesión no se ubica entre los primeros objetivos de la mayoría de estos pueblos.

\subsubsection{El derecho de autodeterminación y la soberanía indígena}

El derecho a la autodeterminación y cualquiera de sus ejecuciones en la práctica en

10 Art. 46 de la Declaración de Derechos de los Pueblos Indígenas de 13 de septiembre de 2007, A/ RES/61/295. 
términos de autonomía para los pueblos indígenas debe ser comprendido siempre como la segunda de las ironías del artículo reseñado previamente (Glenn, 2011), que expresa cómo el idioma de la Declaración es un idioma de derecho puramente occidental, en el que los pueblos indígenas y sus respectivos idiomas han debido comunicarse para poder reivindicar en el ámbito internacional lo que no era posible en otras instancias administrativas más cercanas.

En ese sentido se dibuja la obsesión de los Estados por dejar siempre a un lado la posibilidad de secesión y la escasa intención en este sentido de los pueblos indígenas que si bien pretenden una autodeterminación sin parches, no tienen como uno de sus fundamentos principales la consecución de la secesión ${ }^{11}$.

En este sentido, el relator Anaya habla de que la unión establecida entre libre determinación y el Estado independiente es producto de una comprensión errónea de los instrumentos normativos utilizados para la descolonización africana en el siglo XX así como otros pueblos, para afirmar que el derecho de libre determinación y la Declaración, en cuanto a la manera de desarrollar este derecho, tiene una sustancia reparadora sui generis, así como sería también sui generis la violación del mismo tipo que han venido sufriendo estos pueblos desde tiempos de las colonias hasta nuestros días (Anaya, 2011, p. 47-59).

La cuestión, por tanto, recaería también en dilucidar la posibilidad de convivencia de la soberanía en cuanto concepto clásico estatal y a través del cual se han formulado el derecho y el sistema internacional y la soberanía indígena ${ }^{12}$. Esta formulación de la convivencia es lo que se ha tratado de realizar tanto en el Convenio 169 de la OIT como en la Declaración con el objetivo de que cada estado posteriormente lo adapte en su derecho doméstico para posibilitar una convivencia en términos de multiculturalidad ${ }^{13} \mathrm{o}$ interculturalidad, pero de ninguna forma en cuanto la versión clásica de soberanía equivalente al estado nación emanada a raíz de la Paz de Westfalia.

El contenido de la Declaración en referencia a la libre determinación aparece en los artículos 3 y 4, en el primero como posibilidad de determinar libremente su condición política y perseguir su desarrollo a su manera mientras que el segundo lo expone como lo que en la academia se ha conocido como autodeterminación interna en el sentido de tener la capacidad de autonomía y el autogobierno para las cuestiones

$\overline{11}$ Es posible que algunos pueblos indígenas tengan este objetivo como fundamento o uno más de los suyos, pero, a tenor de sus expresiones en instancias internacionales, no se puede afirmar que sea un sentir mayoritario.

12 Sobre el progresivo desarrollo del indigenismo hasta la soberanía indígena, véase Wiessner (2008, p.1141-1176).

13 Sobre la OIT y su llegada al multiculturalismo, Rodríguez-Piñero (2005b). 
relacionadas con sus asuntos internos y locales. En el Convenio 169 de la OIT no viene reconocido como tal, pero a lo largo de todo el Convenio vienen reconocidos de forma similar a la Declaración en términos de autonomía, si bien se ubica al Estado como destinatario de una mayor cantidad de deberes con relación al cumplimiento de derechos de los pueblos indígenas mientras que en la Declaración se les empodera en cuanto poseedores de estos derechos de manera más clara.

\subsubsection{Pueblos indígenas y subjetividad jurídica internacional}

Otro de los grandes temas bastante controvertidos entre los académicos y que guarda directa relación causa-consecuencia con la conceptualización de los términos pueblos indígenas y el derecho a la libre determinación, es afirmar si los pueblos indígenas tienen o no personalidad jurídica internacional, a raíz de su participación y reconocimiento en los instrumentos estudiados previamente como pueblos indígenas con derecho a la autodeterminación.

El reconocimiento formal de esta personalidad jurídica supondría comprender en clave de igualdad a estos pueblos en comparación con los Estados, que hasta el momento son los únicos poseedores de esta condición en su versión absoluta.

Uno de los grandes motivos de esta discusión es que debido a que la conformación de la institucionalidad internacional no fue producto de un acuerdo en un determinado momento que planteara la mayor cantidad de hipótesis prospectivas de cómo iban a regirse los distintos actores que fueran surgiendo, sino más bien una constante construcción en respuesta ante los respectivos retos que fueron surgiendo en las relaciones internacionales, ha propiciado que no haya un órgano o ninguna otra manera definida de dilucidar quién tiene personalidad legal más allá de la práctica del principal actor que son los mismos Estados ${ }^{14}$.

La práctica y la participación de los Estados en relación con los pueblos indígenas supondrán un reconocimiento de su subjetividad internacional que será simultáneamente el ejercicio del derecho de autodeterminación indígena en sus diferentes esferas de ejecución. Natalia Álvarez Molinero (2009, p. 219) afirma que el reconocimiento en clave de autonomía y con una cierta concepción participativa puede implicar que se pueda ser sujeto del derecho de autodeterminación sin ser sujeto de derecho internacional.

Al continuar siendo el Estado el que decide a quien acepta como sujeto de derecho internacional, nos encontramos con un reconocimiento para los pueblos indígenas

14 Al respecto de si las empresas pueden o no ser sujeto de derechos, véanse Kinley \& Chambers (2006, p. 447-497). 
sin duda superior al que se pueda otorgar a otros actores no estatales como pueden ser las empresas u ONG, pero de ningún modo equivalente al que otorgan a otros Estados, que supone un reconocimiento pleno. Ello supone, a su vez, que el reconocimiento de la validez de los discursos recae solo sobre los Estados y no sobre los pueblos indígenas que son de alguna forma todavía poco más que unos invitados en un sistema ajeno (Kinley \& Chambers, 2006).

Sobre esta perspectiva nos preguntamos si la lucha indígena se deberá dirigir a un reconocimiento completo de su personalidad jurídico-política en tanto sujetos preexistentes a los Estados o bien abogar por seguir fortaleciendo avances de autodeterminación en su ejecución material práctica que supondría un reconocimiento de su subjetividad indirecta.

\subsubsection{El Foro Permanente para las Cuestiones Indígenas y el Mecanismo de Expertos sobre Derechos de los Pueblos Indígenas}

La sola creación de un Foro donde debatir los principales problemas y reivindicaciones de los asuntos indígenas supuso un gran avance en términos de reconocimiento de estas reivindicaciones, así como la posibilidad de obtener un instrumento internacional que pudiera propiciar la ejecución del resto de reivindicaciones a niveles del Estado en que cada uno de estos pueblos habita, así como en los niveles inferiores de la administración estatal. Si a ello le suma el hecho de que al menos la mitad de los expertos que conforman este Foro son nominados por las organizaciones indígenas (ocho sobre 16) para posterior elección por el presidente del Consejo Económico y Social de las Naciones Unidas (ECOSOC), ello fundó unas expectativas en los pueblos indígenas que con el tiempo parecen haber quedado claramente defraudadas (Tapia, 2011).

Las expectativas defraudadas probablemente en parte sean producto de una mala comprensión o de un deseo de ver más allá de la propia literalidad del mandato que tiene el propio Foro, ya que este mandato consiste en preparar información, prestar asesoramiento y formular recomendaciones o difundir actividades relativas a cuestiones indígenas ${ }^{15}$. Un mandato a todas luces carente de capacidad ejecutoria, más allá de suponer poco más que un lobby indígena dentro del sistema, dependiendo finalmente del deseo de los Estados de propiciar mejoras en la práctica respecto de las reivindicaciones indígenas.

Tal y como expresa Luis Rodríguez-Piñero, este reconocimiento parcial de la participación de los pueblos indígenas en un Foro sobre cuestiones indígenas les

$\overline{15}$ El mandato del Foro en http://www.un.org/esa/socdev/unpfii/es/about_us.html 
hizo comprender que participaban en calidad de pueblos frente a los Estados propiciando una "confusión de objetivos" (2011, p. 229-263) sobre si la participación es el objetivo (que como hemos dicho, el reconocimiento supone un baluarte en términos de reparación entre otros asuntos) o la participación es un medio de obtener otros objetivos históricos en sus reivindicaciones. En lo que respecta a la institucionalidad internacional todo aparenta ser una estrategia de cooptación basada en pequeñas concesiones improductivas que apaguen otras formas de lucha indígena (Corntassel, s.f.). Sin embargo, es innegable que estas pequeñas concesiones acaban produciendo en ocasiones más resultados que los esperados por la parte estatal, aun siendo escasas para la parte indígena mediante la apropiación constante de los mecanismos institucionales.

En lo que respecta al mecanismo de expertos en cuanto órgano adscrito al Consejo de Derechos Humanos y con un mandato de informar en cuestiones técnicas a este órgano sobre los derechos humanos de los pueblos indígenas, comparte los mismos problemas del Foro teniendo además una menor repercusión mediática dentro del indigenismo por su mayor burocratización

\section{Conclusiones}

Una de las conclusiones más claras a raíz del artículo expuesto previamente muestra que la participación indígena ha supuesto una consecuencia imprevista cuando se conformó a partir de la Segunda Guerra Mundial el sistema internacional que progresivamente daría pie al derecho internacional en general y al de los derechos humanos en particular. Ante esta situación de hecho, los pueblos indígenas se fueron organizando y aprovechando las circunstancias que en cada momento les fueron resultando más propicias para reivindicar unas cosmovisiones que se veían permanentemente ocultadas, vilipendiadas y atacadas desde siglos atrás por la visión hegemónica occidental y su sistema liberal de desarrollo.

Ante el empuje de la lucha indígena y de otros actores no estatales, los Estados, antes únicos actores de este sistema, han ido cediendo espacios de participación y protagonismo a estos pueblos en el sistema internacional, limitando en la medida de lo posible que su afectación pueda variar la estructura estatocéntrica del sistema, así como el régimen de los Estados-nación como medio de participar como plenos sujetos jurídicos en el ámbito internacional. Ello lleva a pensar con evidente carga fundamentada que a menudo se les permite e incluso alienta la participación con el objeto de cooptar todo el movimiento indígena internacional y con ello ver apagadas o debilitadas las luchas en los niveles más cercanos al Estado.

Esta participación creciente y con espacios cada vez más amplios ha posibilitado que este nuevo actor llamado pueblos indígenas sea un actor a un nivel superior a 
otros actores no estatales como puedan ser las empresas u otros grupos, que sin embargo está lejos todavía de participar en condiciones de igualdad con los Estados, pese a ser una participación claramente innovadora comparándola con otras análogas a priori.

Ello puede suponer una decepción para los pueblos indígenas en términos de reparación. Sin embargo, observando las motivaciones principales que estos pueblos han tenido a lo largo de su participación en la sociedad internacional, buena parte de sus reivindicaciones se verán satisfechas si logran un adecuado desarrollo de sus cosmovisiones indígenas a través del derecho de autodeterminación, que, como sabemos, es el derecho nuclear sobre el que gravitan los demás. Este desarrollo será posible pese a las cláusulas introducidas por los Estados tanto en el Convenio 169 de la OIT como en la Declaración, con el fin de limitar la posibilidad de sustentar sobre él el temido derecho a la secesión que supondría una herida en el régimen estatocéntrico y de Estados-nación sobre el que se sustenta todo el sistema internacional y en el rechazo a inmiscuirse en cuestiones de soberanía estatal por

parte de entes externos. Ha quedado claro a lo largo de todo el artículo que ni la secesión ni el fin del régimen estatocéntrico es una motivación fundamental para los pueblos indígenas, sin perjuicio de que pudieran verse afectados indirectamente por sus reivindicaciones.

Todo el artículo evidencia además que los pueblos indígenas no van a dejar de moverse en un sistema que no controlan y que no fue pensado para ellos, cuestión que no obsta para que con mayor o menor intención estén propiciando cambios fundamentales en términos de influencia y poder.

\section{Referencias}

Aguilar, G. (2006). La aspiración indígena a la propia identidad. Revista Universuum, 21(1), 106-119. Recuperado de http://www.scielo.cl/scielo.php?pid=S0718$23762006000100007 \&$ script $=$ sci_arttext

Allen, S. (2011). The UN Declaration on the Rights of Indigenous Peoples and the Limits of the International Legal Project. In S. Allen \& A. Xanthaki. Reflections on the UN Declaration on the Rights of Indigenous Peoples, Studies in International Law, vol 30. (pp. 225-259). Oxford: Hart Publishing.

Álvarez, N. (2009). El sujeto indígena y el derecho de autodeterminación en la Declaración de Naciones Unidas sobre Derechos de los Pueblos Indígenas: ¿tienen los pueblos indígenas personalidad jurídica internacional? En N. Álvarez, D. Oliva \& N. Zúñiga. Declaración sobre Derechos de los Pueblos 
Indígenas. Hacia un mundo intercultural y sostenible. (pp.312-333). Madrid: Catarata.

Anaya, J. (2011). El derecho de autodeterminación y la autonomía indígena. En F. Gómez \& S. Ardanaz. La plasmación política de la diversidad. Autonomía y participación política indígena en América Latina. (pp. 47-59). Serie Derechos Humanos vol. 17. Barcelona: Deusto.

Brysk, A. (2007). Globalización de los pueblos indígenas: el rol de la sociedad civil internacional en el siglo XXI. En S. Martí i Puig. Pueblos indígenas y política en América Latina. El reconocimiento de sus derechos y el impacto de sus demandas a inicios del S. XXI. (pp.17-31). Barcelona: Bellaterra.

Corntassel, J. (s.f.). Partnership in action? Indigenous political mobilization and cooptation during the first UN indigenous decade (1995-2004). Human Rights Quaterly (29).

Damman, S. (2010, abril). La pobreza indígena en América Latina y el primer objetivo de desarrollo del milenio. Informe sobre pueblos indígenas, pobreza y desarrollo. En Indigenous Peoples, poverty and development. Retrieved from http://siteresources.worldbank.org/EDUCATION/Resources/2782001121703274255/1439264-1288632678541/Indigenous_peoples_full_ report.pdf

Erueti, A. (2011). The International Labor Organization and the Internalization of the Concept of Indigenous Peoples. In S. Allen \& A. Xanthaki. Reflections on the UN Declaration on the Rights of Indigenous Peoples, Studies in International Law, vol 30. (pp-93-121). Oxford: Hart Publishing.

Glenn, P. (2011). The three ironies of the UN Declaration on the rights of indigenous peoples. In S. Allen \& A. Xanthaki. Reflections on the UN Declaration on the Rights of Indigenous Peoples, Studies in International Law, vol 30. (pp. 171-183). Oxford: Hart Publishing.

Gómez, F. (2009). El derecho de los pueblos indígenas a la reparación por injusticias históricas. En N. Álvarez, D. Oliva \& N. Zúñiga. Declaración sobre Derechos de los Pueblos Indígenas. Hacia un mundo intercultural y sostenible. (pp.157193). Madrid: Catarata.

Gómez, M. (2006). El Convenio 169 de la Organización Internacional del Trabajo. En M. Berraondo (coord.) Pueblos indígenas y derechos humanos. (pp.133153). Bilbao: Universidad de Deusto.

ICHRP International Council on Human Rights. (2006). Policy human rights standards: learning from experience. In International Council on Human 
Rights Policy, Versoix, Suitzerland.

Kempf, I. (2009). Cuando la fuerza irresistible mueve al objeto inamovible: aspectos controvertidos en la negociación de la Declaración sobre Derechos de los Pueblos Indígenas. N. Álvarez, D. Oliva \& N. Zúñiga. Declaración sobre Derechos de los Pueblos Indígenas. Hacia un mundo intercultural y sostenible. (pp.51-65). Madrid: Catarata.

Kinley, D. \& Chambers, R. (2006, Oct.). The UN human rights for corporations: the private implications of public international law. Human Rights Law Review, 6(3), 447-497.

Kuper, A. (2003, June). The return of the native. Current Anthropology, 44 (3), $389-402$.

Kymlicka, W. (2011). Beyond the indigenous/minority dichotomy? In S. Allen \& A. Xanthaki. Reflections on the UN Declaration on the Rights of Indigenous Peoples, Studies in International Law, vol 30. (pp. 183-209). Oxford: Hart Publishing.

Martí i Puig, S. (2007). Emergencia de lo indígena en la arena política: ¿un efecto no deseado de la gobernanza? En S. Martí i Puig. Pueblos indígenas y política en América Latina. El reconocimiento de sus derechos y el impacto de sus demandas a inicios del S. XXI. (pp. 127-149). Barcelona: Bellaterra.

Meron, T. (2000). The humanization of international law. American Journal of International Law, 94(2), 239-278.

Minde, H., Eide, A. \& Ahren, M. (2007). The UN Declaration on the Rights of Indigenous.

Peoples, what made it possible? The work and process beyond the final adoption. Galdu Cala Journal of Indigenous Peoples Rights, (4).

OIT (1989). Convenio 169 de la OIT. Informe sobre el problema de la discriminación contra las poblaciones indígenas, conclusiones, propuestas y recomendaciones, E/CN. Sub.2/1986/7add4.

Palacios, A. \& Bariffi, F. (2007). La discapacidad como una cuestión de derechos humanos.

Una aproximación a la Convención Internacional sobre los Derechos de las Personas con Discapacidad. Madrid: Cinca.

Rensahw, J. \& Wray, N. (2004). Indicadores de pobreza indígena. Washington. Recuperado de http://www.bvsde.paho.org/bvsacd/cd27/pobreza-indigena.pdf 
Rodríguez-Piñero, L. (2011). La participación indígena de los pueblos indígenas: una lectura crítica. En F. Gómez \& S. Ardanaz. La plasmación política de la diversidad. Autonomía y participación política indígenas en América Latina. (pp. 229-263). Barcelona: Deusto.

Rodríguez-Piñero, L. (2005a). Indigenous peoples, post-colonialism and international law: the ILO Regime (1919-1989). Oxford: Oxford University Press.

Rodríguez-Piñero, L. (2005b). La OIT y los pueblos indígenas en el derecho internacional: del colonialismo al multiculturalismo. En Caminos hacia el reconocimiento (pp. 101-140). Barcelona: Servei de Publicacions.

Tapia, A. (2010, enero). Declaración de los Derechos Indígenas en Naciones Unidas: dificultades y posibilidades de implementación para la satisfacción de las reivindicaciones indígenas. Trabajos y Ensayos (11). Recuperado de http:// www.diprriihd.ehu.es/revistadoctorado/n11/Tapia11.pdf

Tapia, A. (2011, enero). Participación indígena en el sistema internacional: retos y obstáculos de una participación obligada. Trabajos y Ensayos, (13). Recuperado de http://www.diprriihd.ehu.es/revistadoctorado/n13/ Asier_Tapia13.pdf

UN (2007, 13 de sep.). Declaración sobre Derechos de los Pueblos Indígenas. Recuperado de http://www.un.org/esa/socdev/unpfii/documents/ DRIPS_es.pdf

UN (2008). Resource kit on indigenous people's issues. Retrieved from http:// www.un.org/esa/socdev/unpfii/es/history.html

Wiessner, S. (2008, Oct.). Indigenous sovereignty: reassessment in light of the UN Declaration on the Rights of Indigenous Peoples. Vanderbilt Journal of Transnational Law, 41(4), 1141-1176. 